\title{
CAMBIO ORGANIZACIONAL EN ESTACIONES DE SERVICIO: IMPLICACIONES DE LA REFORMA ENERGÉTICA
}

\author{
ORGANIZATIONAL CHANGE IN SERVICE STATIONS: IMPLICATIONS OF \\ ENERGY REFORM
}

\begin{abstract}
Graziella Guadalupe Ramírez-Méndez $^{1}$ (iD) ; Carlos Alberto Rodríguez-Garza ${ }^{2}$ (D) ; Jorge Alberto Rosas-Castro ${ }^{3}$
1.Maestra en Administración y Dirección Estratégica. Coordinadora de Mercadotecnia en Farmacias del Ahorro. Plaza

Villahermosa. Docente Universidad de Negocios de Tabasco. graziellarmz@hotmail.com.

2. Doctor en Estudios Organizacionales. Universidad Juárez Autónoma de Tabasco, División Académica de Ciencias Económico Administrativas. crogarza@hotmail.com.

3. Doctor en Administración. Universidad Juárez Autónoma de Tabasco División Académica de Ciencias Económico Administrativas.jrosas@hotmail.com.
\end{abstract}

*Correspondencia del Autor: Carlos Alberto Rodríguez Garza, correo electrónico: crogarza@hotmail.com.

\section{RESUMEN:}

Objetivo: Analizar el proceso de adaptación al nuevo modelo de negocio permiso competencia implementada por la Reforma Energética en dos compañías gasolineras, para el reconocimiento de su capacidad de cambio, los riesgos y su resistencia al mismo. Método: Se desarrolló un estudio cualitativo con diseño fenomenológico para descubrir cómo ha sido el ajuste al cambio de modelo. Resultados: En las capacitaciones constantes que se brinda al personal, se ha permitido una integración adecuada en la forma de trabajar y brindar el servicio a los usuarios. Limitaciones: Se presentan resultados limitados al no contar con las experiencias de otras compañías que decidieron no participar en el estudio. Originalidad: Aporta una visión de cambio desde la experiencia de los actores sobre una visión de mercado. Conclusiones: La Reforma Energética dio paso a la oportunidad de competencia de las compañías, lo que provocó buscar las iniciativas de cambio que favorecieran a cada una de ellas.

Palabras clave: Cambio organizacional; reforma energética; nuevo modelo de negocio; Villahermosa; Tabasco.

Cómo citar:

Ramírez-Méndez, Graziella Guadalupe; Rodríguez-Garza, Carlos Alberto; Rosas-Castro, Jorge Alberto. (2020). Cambio organizacional en estaciones de servicio: implicaciones de la reforma energética. Revista de Investigaciones Universidad del Quindio, 32(2), 59-76. https://doi. org/10.33975/riuq.vol32n2.456 


\section{ABSTRACT:}

Objective: To analyze the process of adaptation to the new business model, permit competition implemented by the Energy Reform in two gas station companies, for the recognition of their capacity to change, risks and their resistance to it. Method: A qualitative study was developed with a phenomenological design to discover how the adjustment to the change of model has been. Results: In the continuing staff training, it has been possible to carry out a correct combination of the work done and the service provided to the users. Limitations: These results are limited by not having the experiences of other companies that decided not to participate in the study. Originality: Provides a vision of change from the actors' experience of a market vision. Conclusions: Energy Reform gave way to companies' opportunity for competition, which led to the search for change initiatives that favor each of them.

Keywords: Organizational change; energy reform; new business model; Villahermosa; Tabasco.

\section{Clasificación JEL: M10, M20, Q41.}

\section{INTRODUCCIÓN}

La industria energética mexicana ha ido evolucionando y conforme pasa el tiempo la participación del sector privado en la industria de gas y petróleo se ha ido limitando debido a la fuerte competencia existente.

La Reforma Energética surge del estudio y valoración de las distintas iniciativas presentadas por los partidos políticos representados en el Congreso, debido a una evaluación de desempeño de la industria de hidrocarburos y la necesidad de tener un control de la misma, (Diario Oficial de la Federación, 2013). Fue aprobada por el Congreso de la Unión para determinar un rumbo de modernización para el sector de hidrocarburos de México, tomando en cuenta las empresas públicas que se dedican a prestar sus servicios en este sector. El 20 de diciembre fue publicada la Reforma Constitucional la cual desde su inicio marcó distintos objetivos, entre ellos, mayor autonomía ante el sector, tener empresas productivas, modernizar y fortalecer las actividades, evitar riesgos financieros, geológicos y ambientales, proponer alternativas de contingencia, manejar estándares internacionales de eficiencia, calidad y confiabilidad de suministros, desaparecer la corrupción en el sector y fortalecer la administración de ingresos petroleros (Secretaria de Energía, 2013).

La Reforma representó y representa un cambio de paradigma en la gestión de los recursos naturales de México y en la administración interna y externa de las empresas, representando de manera directa a las empresas privadas dedicas a trabajar en este sector.

La aprobación de la Reforma Energética dio paso a diversos cambios a nivel nacional de la comercialización de combustible a través de nuevas marcas de estaciones de servicio, la sectorización del antes llamado monopolio de PEMEX dio apertura en México a diversas compañías con nuevas e innovadoras estaciones y también petroleras mundiales que entraron al mercado.

Antes de la Reforma Energética, PEMEX y sus organismos subsidiarios tenían a su cargo de manera exclusiva, la venta, la exploración, explotación, refinación y almacenamiento de gasolina y otros combustibles, (IPSOS, 2017).

La venta al público estaba permitida solamente 
a través de un modelo de franquicia-concesión PEMEX, como resultado de la Reforma Energética, a partir de 2017 diferentes empresas (nacionales y extranjeras) pudieron abrir estaciones de servicio, con sus propias marcas, estándares de servicio, manejando un nuevo modelo de negocio llamado permiso competencia, (IPSOS, 2017).

Orellana (2018) señaló que los consumibles se consideran como producto de abasto indispensable, la cual deben de ser subsidiados, como parte de los elementos de la asistencia social y no únicamente como parte del desarrollo económico.

Las gasolineras no contaban con un diseño de rentabilidad propia o diseño de control, solo se trataba de extensiones de una distribución con márgenes garantizados desde la exclusividad, la no competencia y los precios oficiales. (Orellana, 2018).

"Sólo el Estado, personificado en PEMEX, decidiría a quién, dónde y cuándo otorgar una concesión gasolinera. No había un proceso abierto, exigible y transparente para ello, sino la decisión espontánea y graciosa del Estado, que podía otorgar y revocar esas concesiones que llevaban en el trasfondo la propiedad originaria e imprescriptible de la nación respecto de todo hidrocarburo dentro del país" (Orellana, 2018, pág. 1). De igual manera afirma que el contraste del régimen que entró con la Reforma Energética fue la transparencia y no discrecionalidad.

La Comisión Reguladora de Energía (CRE) es la autoridad que otorga los permisos para instalar y operar gasolineras. Su procedimiento está previsto en la Ley de Hidrocarburos, en el Reglamento del Título Tercero de esa Ley, así como en las disposiciones administrativas de la propia CRE, que detallan formatos y trámites. Ese proceso es transparente, abierto y exigible. Cumpliendo los requisitos el permiso debe ser otorgado sin necesidad de voluntad adicional por parte del Estado. El diseño, la operación y el mantenimiento de las gasolineras no requieren del visto bueno del Estado a través de PEMEX, sino de dictámenes de conformidad de mercado abierto elaborados por unidades de verificación también en proceso de ampliar sus capacidades y mercado, (Orellana, 2018).

Actualmente se reconoce la gran actividad empresarial que realizan las estaciones de servicio al ofrecer un producto y servicio el cual es de vital importancia para los ciudadanos, sin embargo, con el paso del tiempo, la actividad de distribución de combustibles ha llegado a darse de manera ilegal, justificando la causa como falta de oportunidad de trabajo en el país, en donde los precios de la gasolina cada día aumentan más y es difícil comprarlos, ya que no hay un sustento económico estable. De acuerdo con datos obtenidos de Transparencia que cita el periódico El Universal; localizó 23 mil 237 tomas clandestinas de Petróleos Mexicanos (Pemex) en el país, del primero de enero 2007 al 31 de diciembre del 2016. Estos datos hasta el 2019 fueron incrementando, creando así una amenaza para el mercado competitivo de las estaciones de servicio, el cual el reto de hoy es, ofrecer un buen producto y servicio con un valor agregado, donde les genere seguridad a los consumidores y la pueden obtener a un precio accesible, con el objetivo de no caer en la distribución y venta ilegal de los combustibles y principalmente prevenir accidentes en los establecimientos donde se realiza esta práctica ilegal (Estrada, 2017).

La Reforma Energética permitió la entrada a nuevas compañías al mercado de competencia en Villahermosa, Tabasco, como lo fue Gasolineras Grupo DG a través de su alianza estratégica con la compañía inglesa British Petroleum(BP), de igual forma la compañía G-500 fue una de las marcas que entró con fuerza en el mercado, cabe señalar que estas compañías se seleccionaron por su gran crecimiento en el estado de Tabasco, a partir del cambio de modelo de negocio, es por ello que el presente estudio se basó en el análisis del proceso de adaptación de las estaciones de servicio de las 
compañías antes mencionadas, la investigación se realizó del 2017 al 2018, analizando los departamentos Mercadotecnia y Administración de cada una de las organizaciones, se hace hincapié que Gasolineras Grupo DG manejaba diversas estaciones de servicio desde antes de la llegada de la Reforma Energética a través de sus empresas Energía DG y Olmeca DG, y su estructura organizacional estaba gestionada $\mathrm{y}$ regulada con el modelo de negocio de franquiciaconcesión que manejaba PEMEX, la compañía G-500 es una empresa mexicana que nació a partir de la alianza del grupo gasolinero G-500 Network y Glencore, multinacional Suiza, líder en distribución de hidrocarburos. Con la apertura del libre comercio de los combustibles dichas empresas han transformaron diversas estaciones de servicio de PEMEX al nuevo modelo de negocio y a su estructura organizacional, de igual manera se han expandido con estaciones nuevas de servicios.

El cambio establecido en la industria de hidrocarburos, principalmente en el sector de las estaciones de servicio ha sido de gran importancia en el mercado y un tema de relevancia en la sociedad, es por ello que el análisis del cambio organizacional de las estaciones de servicio en los departamentos de Mercadotecnia y Administración permitió la identificación de las fortalezas y debilidades y los riesgos que surgieron durante todo el proceso de adaptación al nuevo modelo de negocio, desde la perspectiva individual hasta la perspectiva organizacional, es así como se identificaron estrategias que beneficiaron a la comercialización y posicionamiento de las marcas de estaciones de servicio y la implementación de innovaciones, todo esto a partir del individuo hasta llegar a los objetivos de la organización.

Aunado a lo anterior, se realizaron investigaciones exhaustivas sobre la teoría de cambio organizacional, en donde se hace mención a Newton (2013) el cual expresa que el cambio tiene dos perspectivas de análisis; la humana y la organizacional, por lo que es importante conocer que todo cambio implica una adaptación y que durante esa transición todos los individuos pertenecientes a este ambiente organizacional deben involucrarse, sin embargo, este mismo hecho provoca una resistencia al cambio por lo que en estos elementos recae dicha problemática para poder hacer un estudio y análisis exhaustivo del fenómeno presentado en las estaciones de servicio de cada una de las empresas anteriormente mencionadas.

De acuerdo a lo anterior, se presentaron las siguientes preguntas de investigación ¿Cómo fue el proceso de adaptación al nuevo modelo de negocio permiso competencia implementada por la Reforma Energética en las estaciones de servicio de las compañías Gasolineras Grupo DG y G-500 Network para reconocimiento de sus iniciativas de capacidad de cambio, los riesgos durante la transición y su resistencia al mismo? ¿Cuáles fueron las iniciativas para la gestión del cambio organizacional implementado en las empresas Gasolineras Grupo DG y G-500 Network para la adaptación del modelo de franquicia a permiso competencia? ¿Cuáles fueron las herramientas y técnicas que apoyaron a la planeación y logro del cambio organizacional en las estaciones de servicio de las Gasolineras Grupo DG y G-500 Network? ¿Cuáles fueron los riesgos presentados en la implementación y ejecución del cambio organizacional en cada una de las empresas?

Por lo que el presente estudio se presenta para analizar el proceso de adaptación al nuevo modelo de negocio permiso competencia implementada por la Reforma Energética en las estaciones de servicio de las compañías Gasolineras Grupo DG y G-500 Network para el reconocimiento de sus iniciativas de capacidad de cambio, los riesgos durante la transición y su resistencia al mismo.

A través de identificar las iniciativas para la gestión del cambio organizacional implementado en las empresas Gasolineras Grupo DG y G-500 para la adaptación del modelo de franquicia a permiso competencia y conocer los riesgos 
presentados en la implementación y ejecución del cambio organizacional en cada una de las empresas.

\section{Revisión literaria}

De acuerdo a Acosta y Carlos (2002) las organizaciones cambian debido a que tienen que responder a necesidades y exigencias que permiten su propia evolución, un crecimiento planificado y poder estar al nivel de su competencia. Cada una de ellas tienen razones por las cuales tienen que emprender el cambio para su transformación, por su parte Gordon (1997) menciona que el cambio planificado "describe el proceso sistemático para introducir comportamientos, estructura y tecnologías nuevos con el propósito de abordar los problemas y los retos que enfrenta la organización" (p. 665). El autor Palaci (2005) citado por García y Forero (2016) hace una diferencia entre el cambio planeado y no planeado, el primero lo señala como al logro de metas, mediante etapas, es más organizado y tiene mayor control y relación con temas financieros analizando los costos que el cambio genera. El no planeado es el resultado de fuerzas externas de la organización, ya sea cambios de enfoque de objetivos o exigencias del mercado, involucra innovaciones en sus procesos, en los productos que ofrece, de igual manera analiza los cambios estructurales de la empresa, desde dirección hasta personal.

Los cambios tienen una temporalidad, ya sea a corto plazo o largo plazo, pero una organización no siempre puede ser la misma, requiere de los elementos de adaptación para que esta misma se mantenga y crezca. Acosta y Carlos (2002) definen entonces el cambio organizacional como las distintas transformaciones que se realizan en las diversas áreas de la organización la cual es provocada por fuerzas naturales internas y externas de la organización que surge por la voluntad de quienes las crean y las impulsan.

García y Forero (2016) señalan que el cambio organizacional no es un elemento aislado de lo que pase internamente en la organización, sino debe ser visto como un elemento dinámico que permite ser abordado desde diferentes aspectos del comportamiento organizacional en sus tres niveles (individuo, grupo y organización).

Por otro lado, Newton (2013) comenta que la implementación del cambio implica únicamente que el personal de la organización trabaje de una manera nueva, desde una perspectiva empresarial $\mathrm{y}$ desde un punto de vista a nivel personal.

Una característica del cambio organizacional es cuando se desea aplicar en la organización, no solo se debe de centrar en el individuo de forma aislada, sino en éste dentro de su ambiente social; teniendo como unidad de cambio al grupo social al que el sujeto pertenece.

Lewin (1951) desarrolla una teoría dinámica, donde explica que la conducta del sujeto está en función de la interacción del individuo con su ambiente social, esencialmente en función de los distintos grupos a los que la persona pertenece, señala que el statu quo de la organización es un estado de equilibrio dinámico entre fuerzas que tienden a favorecerlo y fuerzas que tienden a contrarrestarlo o impedirlo; y para que se efectúe un cambio se hace necesario romper con ese equilibrio dinámico entre dichas fuerzas.

Por lo que para Lewin el cambio organizacional inicia con una apertura mental de las personas que trabajan en la organización, el cambio es individual, solo las personas cambian sus habilidades, conductas y relaciones, y solo pueden hacerlo en el trabajo real que desempeñan, nadie más dentro de la organización puede tomar la responsabilidad de cambio de una persona.

Es por ello que cualquier cambio se inicia con la construcción de un modelo organizacional enfocado en los resultados del desempeño de toda la organización; un modelo que podrá después siempre ser afinado, pero que sirve para asegurar que cada colaboradora y colaborador sepa porqué su desempeño y cambio es importante para el 
propósito de toda organización. (Lewin, 1951).

\section{Cambio organizacional desde la Teoría de la Organización}

Romero, Matamoros y Campo (2013) citan a Akhtar y Khan (2011) los cuales señalan que una organización que aprende es adaptativa, de igual manera citan a Bennett y O'Brien (1994) mencionando estos autores que si una organización aprende, facilita el cambio y de igual manera hacen referencia a los autores Recardo, Molloy, y Pellegrino, (1995) siguiendo la idea base y complementándola, si una organización aprende es una organización que cambia.

El cambio organizacional surge de las reacciones externas e internas del ambiente de una organización, para autores como Kast y Rosensweig (1988) citado por Rosas (2008) la complejidad de esta misma depende de las tendenciasdelasociedad, laglobalización, factores macroambientales como el político, económico y social, y a su vez los factores microambientales, la naturaleza interna de la organización, estos elementos determinan la transformación de la organización, las modificaciones estructurales que integran el cambio organizacional y elementos institucionales que lo conforman, reglas, planes y programas. Mapén, Rosas y Rodríguez (2015) citan a Goodman y Kurke, (1982) "El cambio se mira como un proceso secuencial y racional, tendiente a que la organización mejore su eficiencia o desempeño" El proceso incluye de los siguientes elementos: 1. La iniciativa este elemento considera la objetividad de un diagnóstico oportuno de la situación que uno o varios actores o agentes de cambio realicen de manera voluntaria. 2. Se identifican las áreas de oportunidad que la organización tenga, definidos como "problemas". 3. Se hace el diseño de las líneas de acción para la resolución de las áreas de oportunidad identificadas, instrumentando algunas de las posibles alternativas de solución. Y por último se aplican las líneas de acción para la resolución de los problemas y se hace un seguimiento de los resultados.
El cambio organizacional de acuerdo a Burke (2018) y Lines (2005) es un cambio planificado en la estructura formal integrando sistemas, procesos o dominio mercado-producto de una organización, con el objetivo de mejorar el logro de uno o más metas organizacionales. Integra un proceso en el cual un antiguo estado es dejado atrás y una nueva realidad emerge, es posible preguntarse sobre su funcionalidad como un objeto de actitud. Es decir, la actitud hacia un cambio organizacional es definida como la evaluación total que hace la persona acerca del cambio y constituye una tendencia psicológica que es expresa da por medio de la evaluación del cambio con algún grado positivo o negativo (Petty y Wegener, 1998, citado por Rodríguez y Mladinic, 2016).

Newton (2013) señala que el cambio se puede experimentar desde dos niveles, a nivel individual en la organización y el segundo nivel lo expresa como un periodo de riesgo donde pasan las operaciones, la gestión de la organización. Es decir, la dimensión humana en el cambio: el cambio lo realizan los miembros de la organización, dicho autor nos hace referencia a que los sistemas, procedimientos o herramientas hacen este cambio. Y claro está para que se dé un cambio de manera completa se deben de modificar ambos aspectos, sin embargo, debe ocurrir cuando la gente los adopta, en otras palabras, la gente experimenta el cambio a nivel personal. A partir de ahí inicia el cambio.

\section{Factores del cambio organizacional}

De acuerdo a Newton (2013) uno de los principales factores es la resistencia al cambio es un mecanismo humano presentado de manera normal como defensa ante lo desconocido; se refiere como un componente de cambio inevitable, el cual se dice que si no existe una resistencia, entonces no se cree que el cambio es posible.

El autor Newton (2013) define la resistencia como la forma de comportarse de los empleados 
para desacreditar, retrasar o impedir la ejecución del cambio organizacional, el equipo de trabajo se resiste al cambio porque amenaza sus necesidades de seguridad, interacción social, estatus, competencia o autoestima. Entre otras causas de resistencia o pasividad se encuentran: "La resistencia al cambio constituye una conducta natural del ser humano ante cada situación de cambio, ante cada propuesta diferente, ante todo aquello que dista en alguna medida de nuestros esquemas de pensamiento y de acción vigente. Los obstáculos para el cambio son factores ambientales que dificultan la aceptación y la aplicación del cambio" (Molina, 2000, pág. 52).

Los autores Villavicencio y Ocaña (2017) señalan que uno de los problemas principales que causa la gestión del cambio es la resistencia por parte de los individuos que forman parte del proceso, y todos los integrantes de una organización, la resistencia al cambio lleva consigo diferentes percepciones y modificaciones de actividades, rutina, de competencias adquiridas por acciones y roles nuevos que provocan la transformación de lo conocido a lo desconocido, por lo que implica tiempo y dedicación para adaptar la nueva forma de trabajo.

De acuerdo a Vicenzi, Melo y Fonseca (2011) citado por García y Forero (2016) la gestión del cambio es determinante en la creación de mayor o menor resistencia al mismo. La definen como un desacuerdo organizacional referente al cambio, ya que diversas personas la consideran como un aspecto negativo para la organización, no comprendiendo los cambios, percibiendo incertidumbre frente al proceso.

Cuando surgen cambios dentro de la organización surge incertidumbre de parte de los colaboradores, llegan a sentirse amenazados por el cambio, por lo que se debe contrarrestar la amenaza presentada, si no se trabaja en ello, la organización no tendrá la eficacia y eficiencia laboral; de acuerdo a Koontz y Weihrich (1998) por García y Forero (2016) los involucrados en el cambio, es decir, los miembros de la organización, se resisten al cambio por diversas razones, mencionan que estas razones pueden ser, lo desconocido provoca temor e induce resistencia; el desconocimiento de la razón del cambio también genera resistencia y el cambio también puede dar como resultado una reducción de beneficios o pérdida de poder.

La resistencia se puede reducir con un plan de gestión del cambio, disminuyendo así la incertidumbre. Un factor importante para erradicar esta problemática, es la comunicación, permite aclarar razones o efectos del cambio. McLagan (2003) por García y Forero (2016) expresa que la resistencia y las respuestas emocionales al cambio no son algo negativo si no son una señal para prestar atención a las necesidades Son una señal para prestar atención. Indica el cambio de conducta y comportamiento de los miembros, modificando relaciones, lo que pasara a formar parte de la nueva dirección.

Por otro lado, la adaptación al cambio desde una perspectiva del comportamiento organizacional como indican Aguado y Cernada (2016) es la habilidad de las organizaciones para cambiarse a sí mismas, haciendo frente a los cambios y a las contingencias presentadas en su contexto, es decir, se adaptan al modo que la organización se comporta para saber gestionar los cambios imprevistos con objetivos ya establecidos.

En este proceso de adaptación considera los desafíos actuales por diversidad de productos y servicios, que genera incertidumbre, esa nueva demanda del mercado, requiere información gestionada adecuadamente por todos los involucrados que minimice esa fluctuación y permita establecer estrategias organizacionales que influyan en un mejor desempeño (Vega, Martínez, \& Párga, 2019).

Autores como Uzcátegui (2017) citan a Jaffe y Scott (1997) la cual describe la adaptación mediante un modelo de adaptación al cambio organizacional, denominado la curva del cambio. Este modelo, tiene su origen en los estudios de Elisabeth Kübler, Ross sobre las 6 etapas 
por las que pasan los enfermos terminales; cuando conocen que su muerte es inminente: negación, enojo, depresión, miedo, negociación y aceptación. De este modelo Jaffe y Scott (1997) citado por Uzcátegui (2017) adaptaron el modelo de esta doctora al proceso de cambio organizacional; quedando así conformado por cuatro fases: negación, resistencia, exploración y compromiso.

La adaptación es uno de los principales motivos para que las organizaciones no lleguen a su meta, ni cumplan sus objetivos, en este contexto, en un mercado donde la única constante es el cambio, la falta de adaptación es una de las razones, la curva del cambio se aplica como un modelo que presenta las distintas etapas por las que pasa el capital humano; que es afectado por cualquier proceso de transformación a nivel organizacional. Así mismo continua Uzcátegui (2017) el análisis de la curva del cambio es importante ya que las personas, el capital humano pude entender e identificar sus reacciones emocionales de tal manera que se les facilite la transición y brinde el apoyo que necesiten. Este modelo se diseña con el objetivo de utilizarse en una etapa temprana del cambio, cuando se presenta una resistencia alta o los cambios no son deseados, con el fin de orientarlos hacia una adaptación exitosa.

Por ello, la capacidad de cambio, como mencionan autores como Kerber y Buono (2010) citado por García y Forero (2016) implica un enfoque sistémico el cual permita el desarrollo de la organización, donde se puedan aprovechar las capacidades y habilidades de los individuos, sus opiniones y percepciones respecto a la relación entre el personal de la organización, y conocer el proceso y los equipos de trabajo que se necesiten cambiar para apoyar el cambio.

Newton (2013) define la capacidad de cambio como el nivel de capacidad de una empresa para lograr el cambio en momentos específicos, los niveles de experiencia que tienen el equipo de cambio, desde el gerente y los responsables de los departamentos.
Dentro de estos factores debe de considerarse el proceso mismo del cambio parte desde la más mínima interrelación individuo-organizaciónproducto-cliente, hasta el rediseño o innovación de la manera en la cual se debe dirigir una organización para lograr su permanencia en los mercados y alcanzar competitividad ya sea a nivel local, regional, nacional o internacional (Rivera, 2013).

Cuando se presenta un cambio organizacional en las empresas, la adaptación al mismo implica riesgos si no tiene un enfoque de gestión adecuado, los cuales se presentan durante todo este proceso, Newton (2013) señala diversos riesgos que pueden presentar las organizaciones ante las iniciativas de cambio presentadas: Un cambio cuesta dinero y consume los recursos valiosos y a la vez cuesta tiempo del personal altamente calificado y valioso. Durante el proceso de cambio es importante analizar la resistencia al mismo para cumplir con el objetivo de una gestión adecuada de la adaptación al cambio y la prevención de riesgos y su mitigación.

El cambio organizacional según Rivera (2013) se desarrolla mediante dos etapas: la primera determina el proceso de adopción de la influencia y la segunda el proceso de adaptación a la misma.

Las organizaciones detectan las influencias del medio ambiente y reacciona a través del proceso de adopción, por consiguiente, la organización decide tomar la influencia y modificar algún aspecto (proceso de adaptación) o decide no cambiar y no modificar ningún aspecto de ella, dependiendo si es favorable para la misma.

De acuerdo a Rivera (2013) las organizaciones dinámicas (sistema dinámico) adaptan $\mathrm{y}$ adoptan influencias, a diferencia de la anterior las organizaciones estáticas (sistema estático) permanecen sin modificación alguna en el tiempo.

Estos dos procesos (el de adopción y adaptación) manejan un carácter cíclico, el cual permite que 
las influencias y los posibles cambios en las organizaciones estén representados como un proceso constante (Rivera, 2013).

Y por último la transición, Newton (2013) considera al cambio como transformacional, ya que su objetivo es llegar a un gran número de mejoras en la ejecución de la estrategia de gestión de cambio organizacional.

Por otro lado, Baptista (2008) las organizaciones definen objetivos en los cuales todos los miembros de la organización deben de seguir a través de las tareas que les determinen. Las organizaciones pretenden que todos los miembros sean parte de las metas, tratando de tener un control con las personas autócratas; es así como la transición se caracteriza por un proceso gradual y adaptativo, el cual el éxito de la organización radica en la capacidad de entender el cambio, de las iniciativas de cambio propuestas por los equipos de trabajo para desarrollar mecanismos que permitan el seguimiento del sistema.

Sin importar el tamaño, tipo de industria y mercado, todos los cambios que tengan las organizaciones deben ser progresivos, ya que si un cambio no lleva consigo una transición puede causar impacto y trastorno (Baptista, 2008).

\section{El cambio hacia una administración de mercado}

Las innovaciones en la forma de comercializar $\mathrm{y}$ ofrecer los productos y servicios se crean a partir de las necesidades de los consumidores, si bien, las estaciones de servicio de acuerdo a Sabbagh (2006) se crearon para la evolución de las formas de consumir de los clientes, siendo parte del desarrollo de la sociedad. El automóvil y los desplazamientos en las ciudades tienen un valor importante en el desarrollo de la expansión de las mismas, por lo que las estaciones de servicio han beneficiado a los consumidores a tener la facilidad y la accesibilidad en carretera los hábitos de una ciudad atomizada, veloz y sin horarios.
De esa manera las estaciones de servicio de Grupo DG; 100 por ciento tabasqueño que buscando innovar y mejorar buscó la mejor opción de alianza estratégica con la marca BP la cual cuenta con más de 100 años de experiencia trabajando en el sector energético a nivel mundial y en México cuenta con más de 50 años con la marca de lubricantes Castrol y el grupo G-500 empresa mexicana con alianza del grupo gasolinero mexicano G500 y Glencore, empresa multinacional Suiza, líder mundial en trading, distribución de hidrocarburos y materias primas en todo el mundo, con la misión y visión de ser líderes en distribución y comercialización de hidrocarburos en México, con más de 1400 estaciones de servicio con presencia a nivel nacional, han servicio como objeto de estudio para conocer los factores de cambio a los que se enfrenta esta nueva modalidad de servicio.

\section{MÉTODO}

De acuerdo a la teoría analizada con anterioridad de diversos autores como lo son Newton, Molina y Lewin, pioneros en el tema de cambio organizacional se seleccionó una herramienta para la evolución del cambio organizacional en el objeto de estudio. Con base a los objetivos se realiza el análisis de las iniciativas y variables de cambio organizacional presentadas en la problemática, se requiere conocer la conducta y la aptitud del área gerencial durante este proceso de cambio y su iniciativa ante el mismo, para poder identificar las áreas de oportunidad en las que se debe de trabajar y mejorar.

La identificación de la estructura de cada una de las compañías va a favorecer para el análisis de sus fortalezas y debilidades y así proponer la estructura adecuada para cada una de ellas dependiendo de su capacidad.

Mediante la herramienta de la Evaluación de la Capacidad de Cambio que propone Newton (2013) la cual implica la evaluación de la aptitud y capacidad de los participantes de una organización para emprender un cambio. Se 
analizan los factores internos para identificar las iniciativas de cambio, la adaptación, la transición y la resistencia al cambio ante el nuevo modelo de negocio.

\section{Enfoque de la Investigación}

Debido a la naturaleza de la investigación se optó por un enfoque cualitativo, que de acuerdo a Denzin y Lincoln citado por Rodríguez, Gil, y García (1999) se basa en la realidad del contexto natural, interpretando cada una de las circunstancias y los significados de las personas implicadas.

Se utilizó la medición exhaustiva y controlada con el fin de buscar la certeza del mismo, se estudió la adaptación de las estaciones de servicio al nuevo modelo de negocio de las compañías Gasolineras Grupo DG y G-500 Network las cuales formaron parte de esta transición del modelo de negocio en las estaciones de servicio, el estudio se basa en el análisis del proceso de adaptación de las estaciones de servicio de dichas empresas, desde la perspectiva del cambio organizacional.

Para fines de estudio se utilizó el diseño fenomenológico para lograr explorar, describir y comprender las experiencias de las personas con respecto a un fenómeno y descubrir los elementos en común de tales vivencias
(Hernández, Fernández, \& Baptista, 2014), por lo que el estudio se basa en el análisis del proceso de adaptación de las estaciones de servicio para comprender como ha sido su ajuste al cambio de modelo de franquicia-concesión a permisocompetencia con las modificaciones en la gestión organizacional en cada una de las siguientes áreas funcionales, Mercadotecnia y Administración de las compañías Gasolineras Grupo DG y G-500 Network.

El tipo de investigación seleccionada para objeto de estudio es la fenomenología empírica, la cual se enfoca menos en la interpretación del investigador, su objetivo se enfoca en describir las experiencias de los actores clave de la investigación. Analiza una nueva perspectiva del fenómeno o problema bajo estudio Creswell y Moustakas, (1994) citado por Hernández, Fernández y Baptista (2014). En ese sentido, el estudio se enfocó al análisis de las experiencias de los actores clave, los departamentos mencionado, para comprender y conocer su proceso de adaptación al cambio de modelo de franquicia-concesión a permiso-competencia de las empresas Gasolineras Grupo DG y G-500 Network.

Se definen las categorías y subcategorías analizadas en el instrumento de la investigación.

Tabla 1. Categorías y Subcategorías de Investigación

\begin{tabular}{|c|c|}
\hline Categorías & Subcategorías \\
\hline Resistencia al cambio & $\begin{array}{r}\text { Conductas (Comportamiento) } \\
\text { Eficiencia } \\
\text { Aceptación }\end{array}$ \\
\hline Adaptación & $\begin{array}{r}\text { Riesgos } \\
\text { Negación } \\
\text { Incertidumbre }\end{array}$ \\
\hline Transición & $\begin{array}{r}\text { Iniciativas de cambio } \\
\text { Control } \\
\text { Cambio progresivo }\end{array}$ \\
\hline
\end{tabular}




\section{Instrumentos de recolección}

Para el proceso de la recolección de información se seleccionaron diversas técnicas e instrumentos adecuados al método de estudio de casos, donde se aplicaron entrevistas semiestructuradas y revisión documental.

El enfoque cualitativo seleccionado permite que la recolección de datos sea descriptiva, con base en la información que comparten los actores clave del estudio. Jurgenson (2003) afirma que una entrevista es una conversación que tiene como característica una estructura y un propósito, busca entender la perspectiva de las unidades de análisis, comprendiendo y describiendo los significados de las experiencias. Este instrumento permite conocer de manera abierta la perspectiva de los actores clave mediante la construcción de sus respuestas, su flexibilidad da pauta a mayor adaptación de las necesidades de los informantes. Se aplicaron las entrevistas en los departamentos de Mercadotecnia y Administración de las compañías Gasolineras Grupo DG y G-500 Network.

De igual manera se utilizó la técnica de revisión documental que de acuerdo a Arias (2006) es un proceso cuyo objetivo es la recuperación, análisis, crítica e interpretación de información secundaria, es la búsqueda de datos ya registrados acerca de la problemática, en fuentes documentales, electrónicas, para complementar a la investigación con nuevos conocimientos, delimitando el objeto de estudio y así dar pauta a la construcción de hechos mediante la categorización de las experiencias.

La validez del constructo y del contenido de las entrevistas semiestructuradas se utilizó el método de validez de expertos o face vality (Hernández, Fernández, \& Baptista, 2014), mediante tres expertos en el tema de cambio organizacional, quienes realizaron las observaciones y comentarios pertinentes para el ajuste adecuado del instrumento y así tener la legitimidad para la aplicación.

\section{Recolección de la Información}

A continuación, se presenta el procedimiento de la recolección de la información: El objeto de estudio en nuestra investigación: Gasolineras Grupo DG y G-500 Network.

Para llevar a cabo la investigación se elaboró una entrevista semiestructurada la cual se aplicó a cada una de las compañías G-500 Network y Gasolineras Grupo DG, los actores clave seleccionados fueron el Supervisor de Zona de Tabasco de la Compañía G-500 Network y el Contador Público de las Gasolineras Grupo DG.

La entrevista de G-500 Network se realizó en la oficina de la Compañía G-500, ubicada en la ciudad de Cárdenas, Tabasco, consta de nueve preguntas acerca del proceso de adaptación del nuevo modelo de negocio implementado en la estación de servicio, se respondió de manera descriptiva de acuerdo a la situación presentada, tuvo una duración de aproximadamente una hora y media extendiéndose por la explicación de la información del manual compartido acerca del Desarrollo de Expertos en Gestión de Redes EESS. La entrevista se realizó de manera accesible por parte del entrevistado, respondiendo de manera clara cada uno de los reactivos.

Por lo que concierne de la entrevista de Gasolineras DG, se realizó en las oficinas de Promotora DG, donde se encuentra laborando el contador, alrededor de las 17:00, la entrevista tuvo una duración de aproximadamente 40 minutos, donde el entrevistado tuvo la apertura de responder cada una de las preguntas de manera descriptiva, de acuerdo a la situación.

\section{RESULTADOS}

Se presentarán los resultados por cada una de las estaciones de servicio estudiadas y por cada uno de los departamentos de análisis. 


\section{Compañía G-500 Network}

\section{Departamento de Administración}

La información obtenida de los instrumentos de investigación permiten describir que la compañía G-500 desde sus inicios tuvo diversas iniciativas de cambio que favorecieron al crecimiento $y$ expansión de la marca, este cambio del sector energético le permitió la apertura al mercado de competencia de las estaciones de servicio, a través de la gestión, el cambio organizacional se llevó de manera progresiva para procurar el cumplimiento de los objetivos de manera eficiente con los equipos de trabajo.

La transición y transformación de la estación de servicio a la compañía G-500 se realizó en un lapso de 4 meses dando inicio con capacitaciones constantes al personal que iba a laborar en la gasolinera.

Las estaciones de servicio de G-500 cuentan con una infraestructura flexible que permite el acomodo de los espacios de manera que se adecue a la propiedad donde se realizó la instalación.

El supervisor de zona realiza visitas estructuradas de manera periódica de acuerdo a las estaciones de servicio asignadas, el cual orienta el desarrollo en la realización de la visita EESS G-500, tener conocimiento del estatus e identificar problemas.

De igual manera el libre comercio de la administración de precios de los combustibles en las estaciones de servicio permite orientar los objetivos para que cada gasolinera busque su sustentabilidad a través de la atención del servicio al cliente.

Se describen cada una de las categorías y subcategorías que se tomaron para nuestro estudio para conocer el proceso de adaptación de los departamentos de administración y gerencia cambio a modelo de negocio, de acuerdo a la información obtenida de los instrumentos de investigación.
En relación a la resistencia al cambio; los equipos de trabajo tuvieron una conducta positiva ante la transformación de la marca, debido al nuevo modelo de negocio, se trabajó de manera eficiente en tiempo y forma para la transición a la nueva modalidad de negocio, mediante la supervisión de los responsables de la administración de la transformación y Se determinaron acuerdos en la negociación para la aceptación de la transformación a la marca G-500 debido a los beneficios que traía consigo el cambio de la estación de servicio.

De acuerdo a la adaptación; se consideró como riesgo la no adaptación a las políticas de G-500 por parte del personal, siendo una marca mexicana que realizo una alianza estratégica con una empresa suiza, trajo consigo innovación y nuevas iniciativas de cambio para la estación de servicio y la toma de decisión de la inversión de los gerentes de piso de la estación de servicio, al no permitir la colaboración con la marca, el personal administrativo no presentó actitud negativa ante los cambios que la marca G-500 trajo consigo, tuvieron la disposición de colaborar con la organización y existía desconocimiento del control del personal y de la supervisión adecuada de la transformación de la estación de servicio a la marca G-500 y que esta trajera beneficios.

Los resultados de la transición, tomaron decisiones para la integración de los equipos de trabajo a través de un organigrama de la estación de servicio y su definición del puesto. De igual manera capacitaciones constantes para todo el personal de la estación de servicio y poder tener el sentido de pertenencia con la marca, a través de visitas estructuradas de los supervisores de zona se lleva un control de la transición y transformación de la estación de servicio y del personal, desde su construcción hasta el cumplimiento de los objetivos de la empresa y se ha percibido un cambio constante mediante etapas de transición, el personal se percibe motivado en la atención al cliente y el trabajo en equipo desde el inicio de la prospección de la estación de servicio que tendrá el cambio de la marca hasta su primera 
operación.

\section{Departamento de Mercadotecnia}

Con la llegada del nuevo modelo de negocio y la apertura del mercado de las estaciones de servicio, cada una de las compañías que entraron al mercado competitivo, pretenden su posicionamiento orientando sus estrategias de mercadotecnia a la atención al cliente, lo que anteriormente no se percibía.

La compañía G-500 tiene su departamento de Mercadotecnia la cual todas estas herramientas las comparte a los gerentes de las estaciones de servicio para tener una coordinación de la comercialización de combustibles a través de la marca G-500.

En cuanto a las categorías de estudio, en el rubro de resistencia al cambio; el personal responsable del área de mercadotecnia ha tenido un comportamiento positivo donde a través iniciativas de cambio propuestas para la forma de comercializar el producto y brindarle un servicio de calidad a los usuarios. Mediante el uso de las herramientas de mercadotecnia, el personal de los equipos de trabajo ha respondido de manera eficiente el cambio de la marca preparándose en la parte mercadológica y atención al cliente, cumpliendo con los objetivos y el posicionamiento de la marca y los equipos de trabajo han participado en las capacitaciones constantes sobre la atención al cliente, aceptando las políticas y lineamientos que tiene la compañía G-500 y de igual manera a través de sus programas de incentivos para sus colaboradores.

En la adaptación se presentaron riesgos de desconocimiento de la marca y la calidad del producto que se ofrecía por parte de los clientes que ya tenía la estación de servicio y los nuevos clientes al momento de la transformación, se ha percibido una actitud positiva con los empleados con los objetivos de la compañía G-500 que los clientes se vuelvan fieles a la marca, con su participación en las capacitaciones constantes que brinda la parte de mercadotecnia de la compañía y el desconocimiento de que los esfuerzos de mercadotecnia cumplieran con el objetivo del posicionamiento de la marca.

Y la transición en este departamento, provocó que el área de mercadotecnia implementara estrategias para la integración de los equipos de trabajo y que el personal conociera los objetivos de la marca y de la estación de servicio. Se percibe al personal motivado en cumplir con sus responsabilidades laborales, a través de las visitas estructuradas que los supervisores de zona realizan en la estación de servicio, se lleva el control sobre la forma de trabajo con el cliente su percepción acerca de la marca y el cambio de imagen a la marca G-500 tomó aproximadamente 4 meses en la estación de servicio, sin embargo, el mercado cambia de manera constante y la compañía tiene que ir actualizando sus estrategias de mercadotecnia para poder expandirse y mantener su posicionamiento. De igual manera este cambio representa una oportunidad en el mercado con la prospección de la diversificación de sus servicios a través de tiendas de conveniencias y diferentes productos de la marca.

\section{Gasolineras Grupo DG}

\section{Departamento de Administración}

La apertura del sector energético dio entrada a un mercado de competencia, el cual Grupo DG ya tenía conocimiento, por lo que tomó la decisión de hacer la alianza estratégica con la compañía BP para su permanencia y crecimiento en el mercado de gasolineras.

Es importante señalar, que las iniciativas de cambio tomadas por los directivos de esta empresa, han sido beneficiosas para ellos, dándole un valor agregado a la forma de ofrecer el combustible e integrar otros productos como aceites y lubricantes.

De acuerdo a las categorías estudiadas; en resistencia al cambio, la toma de decisiones 
de los directivos de la alianza estratégica con la compañía BP trajo conductas positivas en la organización, ya que fue una estrategia inteligente para poder mantenerse en el mercado de gasolineras y traer consigo innovación, esta alianza estratégica determinó nuevos objetivos para incrementar la eficiencia del personal de los equipos de trabajo y trabajar en conjunto con la compañía BP, orientando sus esfuerzos a una gestión constante de generación resultados y la gestión de la alianza estratégica con la compañía $\mathrm{BP}$, ha tenido gran aceptación por parte del Grupo DG, ya que se han sentido respaldados por la marca a través de su misión y su visión compartida.

En este contexto, en la adaptación se percibió la rotación del personal de los despachadores debido a la inseguridad de la localidad, ya que este puesto se lleva a cabo en tres turnos, mañana, tarde y noche. Este riesgo lo han percibido desde el modelo anterior y permanece como riesgo para la organización, ya que genera costos y tiempo en volver a capacitar al nuevo personal, durante la transformación de las estaciones de servicio pertenecientes a Grupo DG no existió negación por parte de los equipos de trabajo, se tomó de manera positiva, compartiendo iniciativas de cambio, a través del trabajo colaborativo y antes de realizar la alianza estratégica con la compañía BP se analizaron las ventajas y desventajas de colaboración con dicha empresa, sin embargo existía la incertidumbre de cómo iban a responder los colaboradores con la alianza estratégica, los beneficios que traería para el grupo y su apertura en el mercado de estaciones de servicio con los clientes y que los empleados se dieran a la tarea de brindar un servicio al cliente de calidad.

Por su parte, en la transición, se advierte que, a travésun organigrama en cadauna delas estaciones de servicio, se integraron los equipos de trabajo y se determinaron tareas para cada puesto, de igual manera mediante las capacitaciones constantes hacia el personal, se realizó la transición de la estación de servicio con la marca BP, la gestión de la evaluación y control de la transformación se realiza a través de las capacitaciones realizadas por parte de la compañía BP y de igual manera las visitas realizadas por los supervisores de Grupo DG y el cambio de las estaciones de servicio de Grupo DG a la nueva marca BP se realizó en un tiempo de aproximadamente 4 meses, el cual consistió en cambiar totalmente la imagen de la estación de servicio, desde su infraestructura hasta la imagen de los despachadores.

\section{Departamento de Mercadotecnia}

Si bien este cambio de modelo de negocio que trajo la Reforma Energética, se percibió de manera significativa en la forma de comercializar y ofrecer el combustible, ya que la apertura al mercado de competencia de las estaciones de servicio, las compañías ahora se enfocan en su propia sustentabilidad y posicionamiento en el mercado, por lo que el análisis de los cambios en el departamento de Mercadotecnia son de gran importancia para conocer sus iniciativas de cambio ante la adaptación de dicha transición.

Grupo DG se ha ido adaptando a las políticas organizacionales que marca la compañía BP respecto a la parte de Mercadotecnia, debido a la alianza estratégica con British Petroleum (BP) Grupo DG paga un porcentaje para tener el material publicitario de acuerdo a la cantidad de combustible que le suministra BP.

De lo anterior se puede observar la resistencia al cambio de que manera la modificación de la marca se percibió un comportamiento favorable en la organización y los beneficios que la compañía BP trajo para el posicionamiento de las estaciones de servicio y crecimiento de sus utilidades, se evaluó durante el cambio la eficiencia del personal de los equipos de trabajo para el cumplimiento de los objetivos de la organización a través de la percepción de los clientes ante la marca y la atención al cliente brindada y la alianza estrategia con BP y el nuevo modelo de negocio beneficio a la parte mercadológica de las Gasolineras Grupo DG, brindándoles estrategias más eficaces para el posicionamiento de la marca. Generando una 
aceptación positiva en la compañía.

En el sentido de la adaptación; Grupo DG al realizar la alianza estratégica con la compañía BP, percibió el riesgo de que los clientes no asociaban a las estaciones de servicio BP con Grupo DG y provocaba inseguridad en ellos al momento de acudir a la estación de servicio, las iniciativas de cambio hacia la forma de comercializar y promocionar la marca han sido positivas y los equipos de trabajo se han involucrado a las estrategias de mercadotecnia, para brindar un servicio de calidad al cliente y se desconocía la percepción de la marca ante los usuarios y los clientes que ya tenían las estaciones de servicio y los clientes potenciales.

Para terminar con la presentación de resultados, en el factor de transición, mediante las capacitaciones constantes que se brinda al personal, se ha permitido una integración adecuada en la forma de trabajar y brindar el servicio a los usuarios, transmitiendo la misión y visión de la organización, en la parte del control en la transición del cambio de imagen de las estaciones de servicio, se evalúa que se cumplan las políticas y lineamientos de la marca BP y se compartan los logros del posicionamiento de la marca y la transición de la imagen corporativa a la marca $\mathrm{BP}$, representó y representa un cambio constante para la estaciones de servicio de Grupo DG, desde el cambio de imagen de cada una de las gasolineras con las que cuenta el grupo, que abarcó un tiempo de 4 meses aproximadamente, tomando en cuenta la prospección de la apertura de nuevas estaciones de servicio para expandirse en el mercado.

\section{ANÁLISIS Y DISCUSIÓN.}

Al finalizar el análisis del cambio organizacional en las estaciones de servicio en Tabasco y de acuerdo al análisis de los datos de cada una de las compañías que fueron objeto de estudio, se presentan las siguientes conclusiones:

La compañía G-500 Network y Gasolineras
Grupo DG, tuvieron la capacidad de adaptación ante el nuevo modelo de negocio que aperturó la Reforma Energética en el mercado de competencia de las estaciones de servicio, apoyados de la teoría de los autores Kerber y Buono (2010) citados por García y Forero (2016) los cuales mencionan que la capacidad de cambio implica un enfoque que permita el desarrollo de la organización, aprovechando las capacidades y habilidades de los individuos, sus opiniones y percepciones respecto a la relación entre el personal de la organización para realizar el cambio.

G-500 Network y Grupo DG en alianza estratégica con la marca BP, cumplen con la teoría propuesta por Newton (2013) que considera al cambio como transformacional, ya que su objetivo es llegar a un gran número de mejoras en la ejecución de la estrategia de gestión de cambio organizacional, dichas organizaciones implementaron mejoras para el posicionamiento de su marca, con la apertura del libre comercio del combustible, la Reforma Energética dio paso a la oportunidad de competencia de las compañías, lo que provocó buscar las iniciativas de cambio que favorecieran a cada una de ellas y marcar la diferencia mediante el valor agregado adquirido.

G-500 Network Y Gasolineras Grupo DG tuvieron la capacidad de toma de decisión para poder adentrarse en el mercado de gasolineras y adaptarse a las necesidades del mercado, caso contrario dichas empresas no iban a tener un crecimiento en el mercado, es donde podemos señalar la teoría de autores como Carlos y Acosta (2002) quienes señalan que las organizaciones cambian debido a que tienen que responder a necesidades y exigencias que permiten su propia evolución, un crecimiento planificado y poder estar al nivel de su competencia.

De igual manera se reconoce que las iniciativas de cambio son las que motivan a la trasformación organizada y planificadas de las organizaciones, en el caso de las estaciones de servicio G-500 Network y Gasolineras Grupo DG mediante la 
gestión de sus departamentos de administración y mercadotecnia pudieron abrirse paso en el mercado gasolinero, orientando sus objetivos a brindar un servicio de calidad con los usuarios y el valor agregado de sus productos, respondiendo así a las exigencias y necesidades del mercado, haciendo énfasis a lo señalado por Newton (2013) que una iniciativa de cambio la define como un cambio especifico que una organización quiere emprender ante las circunstancias presentadas en su entorno, el cual se estructura como proyecto, un programa, una iniciativa seis sigmas, evaluación de capacidad de cambio durante la transición.

La adaptación al cambio organizacional en cada una de las compañías se realizó con un enfoque positivo, constante y preventivo, tomando en cuenta los factores determinantes para que este cambio se llevara a cabo en beneficio de toda la organización, es así como se cumple la teoría de Aguado (2017) donde la adaptación la señala como la habilidad de las organizaciones para cambiarse a sí mismas, haciendo frente a los cambios y a las contingencias presentadas en su contexto, es decir, se adaptan al modo que la organización se comporta para saber gestionar los cambios imprevistos con los recursos que la empresa tenga, es decir, G-500 y Gasolineras DG durante la transición tuvieron que aprender a gestionar los cambios que traía consigo el nuevo modelo de negocio.

No obstante, es importante considerar los riesgos y las áreas de oportunidad que se identificaron para proponer alternativas de solución y $\mathrm{su}$ evaluación constante para tener mayor capacidad al cambio y estar prevenidos ante la contingencia que se presente.

Con dicho análisis del proceso de adaptación se coincide con la teoría de Newton (2013) de que el cambio se da a través de dos dimensiones, humana y organizacional, dando inicio desde la parte del personal hasta poder llegar a la parte sistemática. De acuerdo a Lewin (1951) el cambio organizacional inicia con una apertura mental de las personas que trabajan en la organización, el cambio es individual, solo las personas cambian sus habilidades, conductas y relaciones, y solo pueden hacerlo en el trabajo real que desempeñan, nadie más dentro de la organización puede tomar la responsabilidad de cambio de una persona. Es importante considerar que el mercado de estaciones de servicio ha sido poco estudiado en lo que compete en la parte de gestión organizacional, y que los cambios y el crecimiento de la competencia en el mercado seguirá de manera constante, por lo que cada una de las empresas deben de estar prevenidas y preparadas para ir adaptándose a los factores que intervengan y que las estaciones de servicio de combustible que hoy en día no se han sumado a la actualización del nuevo modelo de negocio, consideren hacerlo para formar parte del mercado competitivo.

De acuerdo al estudio presentado, aprovechando la experiencia de las compañías analizadas, se puede sugerir un esquema de gestión para la adaptación al cambio organizacional de una estación de servicio al nuevo modelo de negocio permiso-competencia, que permita identificar los componentes de investigación y análisis que se propone para las estaciones de servicio que aun han aplicado el cambio de modelo de negocio de manera homogénea, donde estas mismas, podrán obtener beneficios considerables.

\section{Análisis de los modelos de negocio}

Como primera etapa se debe de hacer un análisis y prospección del modelo anterior de franquicia concesión para conocer cuáles eran sus limitaciones, ventajas y desventajas y posterior el análisis del nuevo modelo de negocio permisocompetencia y así poder determinar los objetivos organizacionales para el cambio progresivo de la estación de servicio.

Durante todo este proceso se determinan los tiempos y etapas de la transición de modelo de negocio.

Línea de adaptación. Resistencia al cambio 
Análisis de la conducta del personal de los colaboradores que participaran en el cambio del modelo de negocio.

Riesgos del cambio

Se analizan las ventajas y desventajas, las áreas de oportunidad y donde será el inicio del cambio de modelo de negocio.

Iniciativas de cambio

En esta etapa se presentan propuestas de crecimiento y mejoras de la organización de los colaboradores.

Aplicación de las iniciativas de cambio

Se evalúan las propuestas presentadas en las iniciativas de cambio, se seleccionan las más viables y se aplican las líneas de acción determinadas. En este punto, ya se aplica el nuevo modelo de negocio para las estaciones de servicio, permiso-competencia, el cual va dependiendo de las características de la compañía.

Evaluación de control y desempeño

Esta última etapa es importante, ya que se evalúa el cumplimento de metas organizacionales y el desempeño del personal, debe de ser constante para identificar las debilidades y fortalezas en las que se debe trabajar.

Se analizan los beneficios y la competencia en el mercado de las estaciones de servicio.

Estos elementos descritos de la línea de adaptación al cambio organizacional, permitirá un cambio progresivo y controlado de las estaciones de servicio, con el objetivo de darle un valor agregado a la forma de vender y ofrecer el combustible y orientar todos sus esfuerzos a la satisfacción en el servicio al cliente.

Figura 1. Esquema de gestión para la adaptación al cambio organizacional de una estación de servicio al nuevo modelo de negocio permiso-competencia

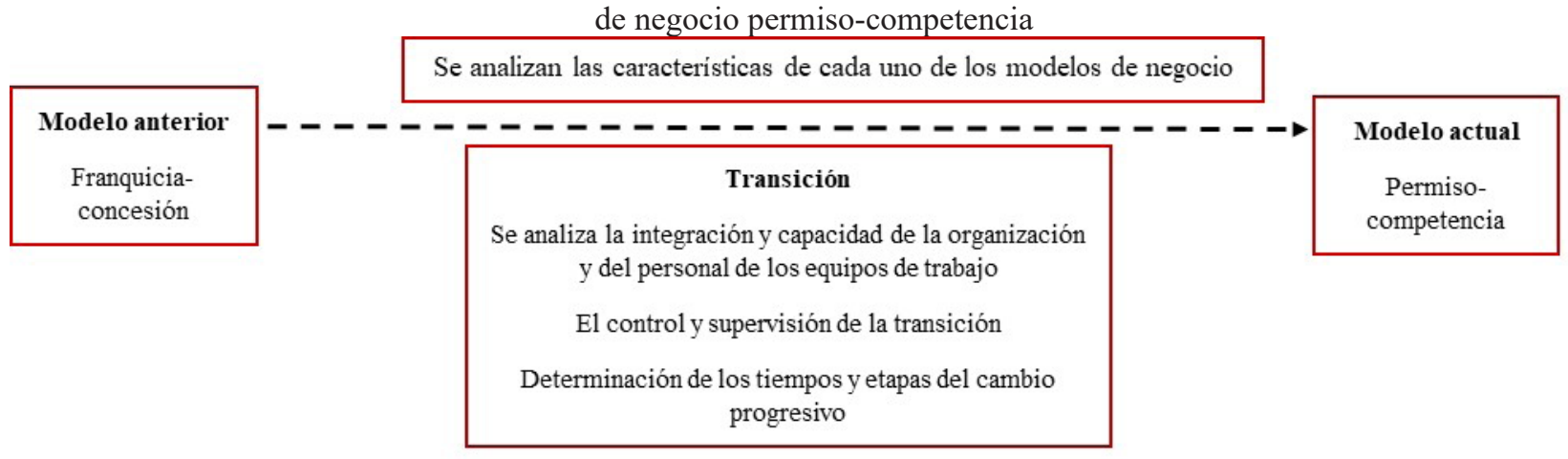

La Línea de Adaptación

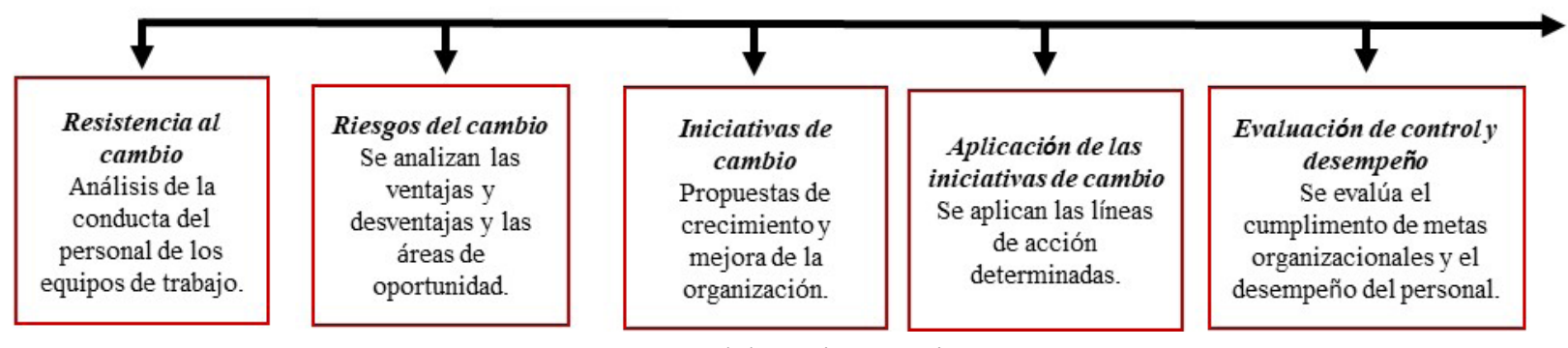

Fuente: Elaboración propia 


\section{REFERENCIAS}

1. Acosta, R., \& Carlos, A. (2002). Cuatro preguntas para iniciarse en cambio organizacional. Revista Colombiana de Psicología, (11), 9-24.

2. Aguado, D., \& Cernada, R. (2016). Adaptarse o morir lentamente. La capacidad de adaptación de las organizaciones como elemento clave de supervivencia. Observatorio de recursos humanos y relaciones laborales, (118). 36-40.

3. Arias, F. (2006). El Proyecto de Investigación, introducción a la metodología científica. Caracas, Venezuela: Episteme, c.a. 5ta edición.

4. Baptista, R. (2008). Cultura: la clave para la transicion organizacional. Punto Cero, (13/16), 85-90.

5. Burke, W. (2018). Organization change: Theory and practice. SAGE.

6. Diario Oficial de la Federación, Decreto (Estados Unidos Mexicanos. 20 de 12 de 2013).

7. Estrada, A. (16 de mayo de 2017). Huachicoleros, reyes de la ordeña. El Universal, págs. https:// www.eluniversal.com.mx/articulo/periodismo-de-investigacion/2017/05/16/huachicoleros-reyes-dela-ordena.

8. Garcia, M., \& Forero, C. (2016). Calidad de Vida Laboral y Disposición al Cambio Organizacional en Funcionarios de Empresas de la Ciudad de Bogotá, Colombia. Acta Colombiana de Psicología, (19/1), 79-90. http://doi.org./10.14718/ACP.2016.19.1.5.

9. Gordon, J. (1997). Comportamiento Organizacional. Prentice Hall.

10. Hernández, R., Fernández, C., \& Baptista, P. (2014). Métodologia de la Investigación. México: Mc Graw Hill.

11. IPSOS. (11 de Agosto de 2017). Reforma Energética: Nuevas Estaciones de Servicio. Ipsos: https:// www.ipsos.com/es-mx/reforma-energetica-nuevas-estaciones-de-servicio

12. Jurgenson, J. L.-G. (2003). Cómo hacer investigación cualitativa, Fundamentos y Meotodología. Ciudad de México: Paidós.

13. Lewin, G. (1951). Fiels theory in social science: Selected theoretical papers. Harper.

14. Lines, R. (2005). The structure and function of attitudes toward organizational change. Human Resource Development Review, (4), 8-32. http://doi.org/10.1177/1534484304273818.

15. Mapén, F. d., Rosas, J. A., \& Rodríguez, C. A. (2015). De la teoría a la práctica: ejercicio de fortalecimiento de la identidad universitaria desde las fronteras interdisciplinarias del cambio organizacional. Revista Forum Doctoral, (6).

16. Molina, H. (2000). El Desarrollo Organizacional como Facilitador de Cambio. Estudios Gerenciales, pp-13-25.

17. Newton, R. (2013). Cambio Organizacional. Trillas.

18. Orellana, A. (25 de Mayo de 2018). Oil \& Gas Magazine. Obtenido de Las Gasolineras, de la franquicia-concesión al permiso-competencia: https://oilandgasmagazine.com.mx/2018/05/lasgasolineras-de-la-franquicia-concesion-al-permiso-competencia/

19. Rivera, Á. (2013). El Cambio Organizacional: Un Proceso de Adopción y Adaptación. Gestión y Estrategia, (44), 93-105.

20. Rodríguex, V., \& Mladinic, A. (2016). Ambivalencia Actitudinal Ante el Cambio Organizacional: Un Análisis Desde el Individuo en Contexto Laboral. Revista Psykhe, (25/1), 1-17. http://doi.org/10.7764/ psykhe.25.1.733 .

21. Rodríguez, G., Gil, J., \& García, E. (1999). Metodología de la Investigación Cualitativa. Ediciones Aljibe.

22. Romero, J., Matamoros, S., \& Campo, C. (2013). Sobre el Cambio Organizacional. Una revisión bibliográfica. INNOVAR Journal, pp. 35-52.

23. Rosas, J. (2008). El cambio en las universidades públicas estatales (UPES) de 1982 a 2004. Estudio de Caso: Universidad Juárez Autónoma de Tabasco. Tesis doctoral. Querétaro, Centro Universitario Querétaro.

24. Sabbagh, J. (2006). Estaciones de Servicio. $A R Q$, (62), 62-65.

25. Secretaria de Energia. (2013). Reforma Energetica. México: Gobierno de la Republica.

26. Uzcátegui, J. (18 de May de 2017). Prozess Group. Obtenido de La curva del cambio: Un modelo para la adaptación organizacional: http://www.prozessgroup.com/la-curva-del-cambio-modelo-laadaptacion-organizacional/

27. Vega, J., Martínez, M. d., \& Párga, N. (2019). Influencia del aprendizaje organizacional y los resultados de las Pymes. Investigación Administrativa, (48) 124.

28. Villavicencio, V., \& Ocaña, I. (2017). El coaching como herramienta para mitigar la resistencia al proceso de cambio organizacional. Palermo Business Review, 83-100. 